\title{
Low Vision Aid for a Patient with the Optic Neuropathy Due to Neuro-Brucellosis
}

\author{
Fatih M Adibelli ${ }^{1^{*}}$ and Deniz Altinbay ${ }^{2}$ \\ ${ }^{1}$ Department of Ophthalmology, Faculty of Medicine, Harran University, Turkey \\ ${ }^{2}$ Niv Eye Center, Adana, Turkey
}

*Corresponding author: Assist. Prof. Fatih Mehmet ADIBELLI, Department of Ophthalmology, Faculty of Medicine, Harran University, Osmanbey Kampusu, 63100 Sanliurfa, Turkey, Tel: +90-414318-3000, Fax: +90-4143183190

\begin{abstract}
Brucellosis is a multisystem infectious disease, which still endemic in Middle East, Mediterranean and South America. Bilateral optic neuritis secondary to the meningeal inflammation is very rare complication of brucellosis. Here we present a 13-year-old male patient with the ocular brucellosis who has optic neuropathy, concluded his treatment and low vision aid for the visual impairment.
\end{abstract}

\section{Keywords}

Low vision aid and rehabilitation, LVA, Neuro-brucellosis

\section{Introduction}

Brucellosis is a multisystem infectious disease, which appear in miscellaneous clinical manifestation. Various organ involvement can be detected in effected person, but the most important ocular complications are anterior uveitis and choroiditis [1]. Besides of this two-condition ocular brucellosis may present with other symptoms such as endophthalmitis, dacryoadenitis, extraocular muscle paralysis, conjunctivitis, episcleritis, nummular keratitis, retinal edema and hemorrhage, retinal detachment, and optic neuropathy. Even brucellosis is endemic some part of the World as in Turkey, but the cranial nerve involvement is a rare condition [2].

In this study, we aimed to present a case with ophthalmoplegia and bilateral optic neuropathy due to brucellosis, which are late complication of brucellosis in a child and managed by low vision aid (LVA).

\section{Case Report}

A 13-year-old male patient with the acute onset of fever and complaints of sweating headache, muscle and joint pain was admitted to the emergency room.

Complete blood count, biochemistry and serology tests was performed. Because of the result of tube agglutination test for brucellosis was $1 / 320$ he immediately hospitalized in pediatric infection unit. Systemic ceftriaxone, rifampin and doxycycline combination therapy was started. Cranial computed tomography scan and magnetic resonance imaging with contrast were unremarkable. The patient was referred to the ophthalmology department because of starting before the left eye (LE), right eye (RE) after the sudden onset of blurred vision, pain around the eyes and double vision. In his ophthalmologic examination best, corrected visual acuity of the RE was 20/400 (logMAR 1.30) and the LE was hand motion (HM). Relative afferent pupil defect was detected on his both eyes. Right and left optic disc showed marked swelling and hyperemia and he had 20-prism diopter of left exotropia.

The patient was diagnosed with optic neuropathy secondary to neurobrucellosis has recommended the continuation of existing treatments.

Six months after in his control exam, BCVA of the RE was 20/200 (logMar 1.0), and LE was finger counting in 2 meters. Right and left optic disc showed pale optic discs (Figure 1). Intraocular pressures were found within normal limits in his both eyes. He had 20 prism diopters of left exotropia (Figure 2).

This case of neurobrucellosis secondary optic atrophy was referred to a low vision aid unit. 1673-1 Keple-

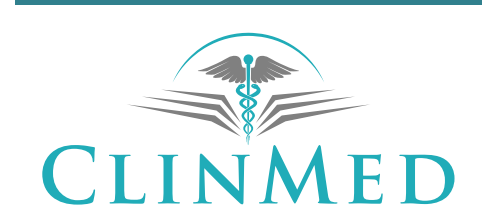

INTERNATIONAL LIBRARY

Citation: Adibelli FM, Altinbay D (2018) Low Vision Aid for a Patient with the Optic Neuropathy Due to Neuro-Brucellosis. Int J Ophthalmol Clin Res 5:096. doi.org/10.23937/2378-346X/1410096

Accepted: November 08, 2018: Published: November 10, 2018

Copyright: (c) 2018 Adibelli FM, et al. This is an open-access article distributed under the terms of the Creative Commons Attribution License, which permits unrestricted use, distribution, and reproduction in any medium, provided the original author and source are credited. 


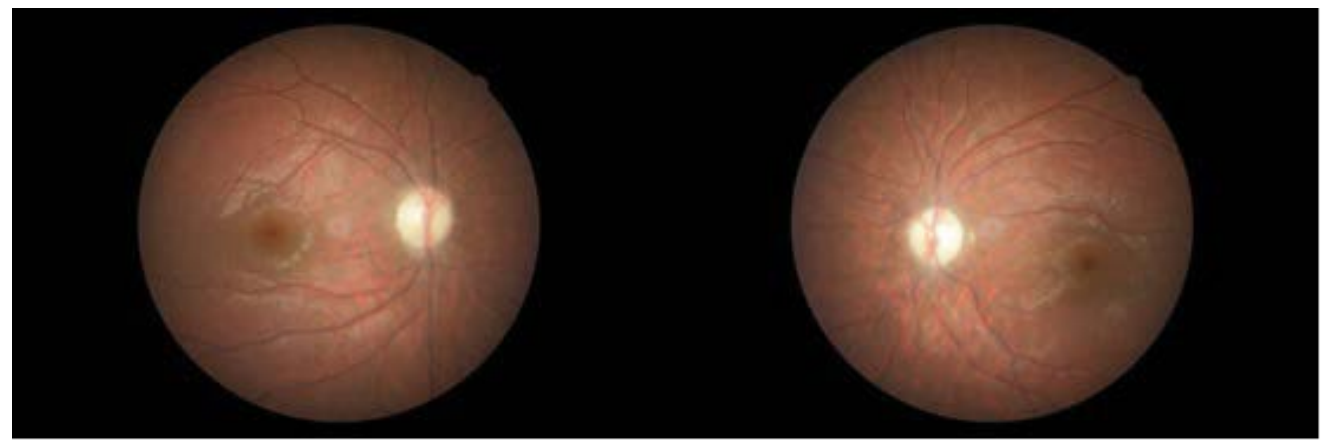

Figure 1: Optic disc appearance of the fundus, during the examination of the low vision aid.

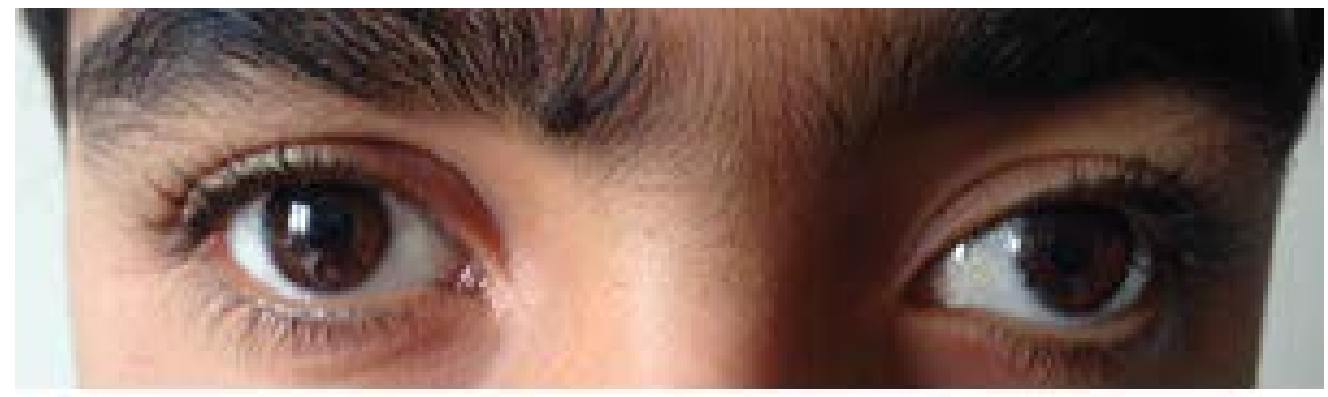

Figure 2: The appearance of left exotropia of the neuro-brucellosis patient.

rian telescopes (Eschenbach, Nürnberg, Germany) had been preferred for improve his visual equity which can provide to magnify the image 2.8 times for distance. By the telescope RE distance visual acuity improved from logMAR 1.0 to logMAR 0.3. The near vision improved from logMAR 1.0 to logMAR 0.3 by using +3.00 cap over the telescope. The patient education was given to the use of telescopic glasses.

\section{Conclusion}

Depending on the habit of eating raw meat or unpasteurized dairy products, brucellosis, it is endemic in some regions as in Turkey. Some of the people, who live in the southern of Turkey, have habit of feeding with raw meat. In our case's family history was positive for the habit of feeding with raw meat.

Dacryoadenitis, conjunctivitis, episcleritis, iridocyclitis, nummular keratitis, cataract, glaucoma, multifocal choroiditis, retinal edema and hemorrhages, exudative retinal detachment, maculopathy and optic neuropathy are among the ocular complications of brucellosis [3]. Meningeal inflammation of the optic nerve involvement in brucellosis cases and secondary axonal degeneration may be due to the current changes in the optic nerve has been considered in a study [4]. Bilateral optic neuritis secondary to brucellosis has been identified that there is a regression in cases of anti-brucellosis and steroid combined therapy giving a positive response to findings [5].

The clinical findings in this patient consisted of optic disc edema, left exotropia and low vision. Systemic ceftriaxone, rifampin and doxycycline combination therapy has been choosing for his therapy. After acute onset was over, we preferred to the Keplerian telescope has been used in the rehabilitation of the patient's vision. Keplerian telescope is a kind of low vision aid system, which is preferred to use in the cases of intermediate, and advanced levels of the low vision. It provides brighter images and wider fields of view [6].

Decreasing the prevalence of brucellosis in developing countries and ocular complications are rare. In endemic areas should be kept in mind in patients with ocular complications in brucellosis, and routine ophthalmologic examination should be performed. The management of the low vision by telescope is helpful in the patients with the optic neuropathy due to neuro-brucellosis.

\section{References}

1. Young EJ (1983) Human brucellosis. Reviews of Infectious Diseases 5: 821-842.

2. Sungur GK, Hazirolan D, Gurbuz Y, Unlu N, Duran S, et al. (2009) Ocular involvement in brucellosis. Can J Ophthalmol 44: 598-601.

3. Karakurum Goksel B, Yerdelen D, Karatas M, Pelit A, Demiroglu YZ, et al. (2006) Abducens nerve palsy and optic neuritis as initial manifestation in brucellosis. Scand J Infect Dis 38: 721-725.

4. Rolando I, Olarte L, Vilchez G, Lluncor M, Otero L, et al. (2008) Ocular manifestations associated with brucellosis: a 26-year experience in Peru. Clin Infect Dis 46: 1338-1345.

5. Abd Elrazak M (1991) Brucella optic neuritis. Arch Intern Med 151: 776-778.

6. Peli E, Vargas-Martin F (2008) In-the-spectacle-lens telescopic device. J Biomed Opt 13: 034027. 